\title{
Irbesartan Ameliorates Diabetic Nephropathy by Suppressing the RANKL-RANK-NF- $\kappa$ B Pathway in Type 2 Diabetic db/db Mice
}

\author{
Xiao-Wen Chen, ${ }^{1}$ Xiao-Yan Du, ${ }^{1}$ Yu-Xian Wang, ${ }^{2}$ Jian-Cheng Wang, \\ Wen-Ting Liu, ${ }^{1}$ Wen-Jing Chen, ${ }^{1}$ Hong-Yu Li, ${ }^{1}$ Fen-Fen Peng, ${ }^{1}$ Zhao-Zhong Xu, ${ }^{3}$ \\ Hong-Xin Niu, ${ }^{1}$ and Hai-Bo Long ${ }^{1}$ \\ ${ }^{1}$ Department of Nephrology, ZhuJiang Hospital, Southern Medical University, Guangzhou 510280, China \\ ${ }^{2}$ Department of Gerontology, ZhuJiang Hospital, Southern Medical University, Guangzhou 510280, China \\ ${ }^{3}$ Department of Emergency, ZhuJiang Hospital, Southern Medical University, Guangzhou 510280, China \\ Correspondence should be addressed to Hai-Bo Long; longhb1966@163.com
}

Received 23 October 2015; Accepted 20 December 2015

Academic Editor: Hannes Neuwirt

Copyright (c) 2016 Xiao-Wen Chen et al. This is an open access article distributed under the Creative Commons Attribution License, which permits unrestricted use, distribution, and reproduction in any medium, provided the original work is properly cited.

\begin{abstract}
The receptor activator of NF- $\kappa \mathrm{B}$ ligand (RANKL) and its receptor RANK are overexpressed in focal segmental glomerular sclerosis (FSGS), IgA nephropathy (IgAN), and membranous nephropathy (MN). However, the expression and the potential roles of RANKL and RANK in diabetic nephropathy (DN) remain unclear. Irbesartan (Irb) has beneficial effects against diabetes-induced renal damage, but its mechanisms are poorly understood. Our present study investigated the effects of Irb in DN and whether the renal protective effects of Irb are mediated by RANKL/RANK and the downstream NF- $\kappa \mathrm{B}$ pathway in $\mathrm{db} / \mathrm{db}$ mice. Our results showed that $\mathrm{db} / \mathrm{db}$ mice revealed severe metabolic abnormalities, renal dysfunction, podocyte injury, and increased MCP-1; these symptoms were reversed by Irb. At the molecular level, RANKL and RANK were overexpressed in the kidneys of $\mathrm{db} / \mathrm{db}$ mice and Irb downregulated RANKL and RANK and inhibited the downstream NF- $\kappa$ B pathway. Our study suggests that Irb can ameliorate DN by suppressing the RANKL-RANK-NF- $\kappa$ B pathway.
\end{abstract}

\section{Introduction}

$\mathrm{DN}$, which is characterized by progressive albuminuria and a gradual decline in glomerular filtration rate, is a distinct kidney disease that is becoming the single most important cause of end-stage renal disease (ESRD) throughout the world [1, 2]. Approximately $30 \%$ of patients with type 1 diabetes mellitus develop DN and approximately 25 to $30 \%$ of patients with type 2 diabetes mellitus (T2DM) will develop overt DN [3, 4]. Diverse pathogenic mechanisms contribute to $\mathrm{DN}$, including advanced glycation end products, activation of protein kinase $\mathrm{C}$, and overexpression of different growth factors. However, accumulating studies suggest that podocyte injury $[5,6]$ and inflammatory processes [7-9] also play vital roles in the development and progression of DN. Podocyte injury has been observed in the kidneys of diabetic patients and animal models and can precede and predict the occurrence of proteinuria $[5,6]$. Additionally, MCP-1, which is the strongest known chemotactic factor for monocytes, is a key inflammatory factor in the pathogenesis and progression of DN $[7,9,10]$. Thus, therapies that protect podocytes from injury and control inflammation are very important to the treatment of DN.

Angiotensin II receptor blocker (ARB) offers effective renoprotection in diabetic animal models and diabetic patients. In animal studies, Irb significantly lowers the blood lipid level and improves the kidney function of diabetic mice and rats $[11,12]$. In clinical trials, Irb and telmisartan are effective in reducing proteinuria in patients with $\mathrm{DN}$ $[13,14]$. Additionally, Irb increases glomerular synaptopodin immunoreactivity, a marker of functional podocytes, and 
exerts a beneficial effect on diabetes-induced renal inflammation [15]. However, the molecular mechanism through which $\mathrm{ARB}$ reverses kidney dysfunction remains incompletely understood.

The RANKL system consists of a triad of proteins: type II membrane protein RANKL, its biological receptor RANK, and decoy receptor osteoprotegerin (OPG). RANKL signaling has been extensively studied in osteoclasts. Binding of RANK initiates a signaling cascade that activates NF- $\kappa \mathrm{B}$ and mitogen-activated protein kinases, which induce osteoclastogenesis and lead to osteoclast-related diseases such as osteoporosis [16, 17]. Recently, Liu et al. [18] identified a new role of RANKL and RANK. They reported that RANKL and RANK were overexpressed in FSGS, IgAN, and MN and were specifically expressed in podocytes. A genome-wide association study also revealed a polymorphism in the podocyte receptor RANK that leads to a decline in renal function in coronary patients [19]. Kiechl et al. found that, as the chief upstreaming molecules of NF- $\kappa \mathrm{B}, \mathrm{RANKL}$ and RANK play a pivotal role in the pathophysiology of T2DM [20]. However, little is known about the potential role of RANKL and RANK in the development of DN.

The present study investigated the role of RANKL and RANK in type $2 \mathrm{DN}$ and whether the renoprotective effects of Irb were mediated by the RANKL-RANK-NF- $\kappa$ B pathway.

\section{Materials and Methods}

2.1. Animal Model and Drug Treatment. Male $\mathrm{db} / \mathrm{db}$ mice in a C57BL/KsJ (BKS.Cg-Dock7m +/+ Leprdb/J) background and their age-matched, normal, wild-type $\mathrm{db} / \mathrm{m}$ littermates were obtained from the Animal Model Research Center of Nanjing University. Mice were housed in the Laboratory Animal Center of Sun Yat-sen University under controlled temperature $\left(20-22^{\circ} \mathrm{C}\right)$, light (alternating 12-hour light/dark cycle), and humidity (50\%-60\%) conditions and received food and water freely. After 1 week of acclimatization, the $\mathrm{db} / \mathrm{db}$ mice were randomly divided into 2 groups $(n=$ 12 each): $\mathrm{db} / \mathrm{db}$ mice and $\mathrm{db} / \mathrm{db}$ mice+Irb (50 mg/kg/day). Additionally, $12 \mathrm{db} / \mathrm{m}$ mice served as the control group. After 12 weeks of Irb treatment, the mice were 20 weeks old and were sacrificed for this experiment. The Animal Care and Use Committee of Sun Yat-sen University reviewed and approved all animal studies and all procedures were in compliance with the National Institutes of Health Guide for Care and Use of Laboratory Animals (Publication number 85-23, revised 1985).

2.2. Serum Measurements. The mice were fasted overnight and then blood was sampled from the eyes of the mice. Serum triglycerides (TG), total cholesterol (TC), blood urea nitrogen (BUN), and serum creatinine (Scr) levels were determined using an automatic analyzer (Roche, Basel, Switzerland). Fasting blood glucose (FBG) was measured using a glucose meter (Roche Diagnostics GmbH, Mannheim, Germany). Fasting insulin (FINS) was measured by an ELISA assay (Jiancheng, Nanjing, China).
2.3. Urinary Albumin to Creatinine Ratio (ACR) Assay. Mice were placed in metabolic cages for urine collection at 8,12 , 16 , and 20 weeks of age. The collected urine specimens were centrifuged at $10,000 \times \mathrm{g}$ for 5 minutes at $4^{\circ} \mathrm{C}$. The microalbumin level in the urine supernatants was measured using a murine microalbuminuria ELISA kit (Exocell, Philadelphia, PA, USA), and the creatinine in the urine was assayed by the Creatinine Companion kit (Exocell, Philadelphia, PA, USA). Then, the ACR was calculated as previously described [21].

2.4. Histological Analysis. Kidneys were cut longitudinally, and the kidney cortexes were fixed with neutral formalin for performing paraffin sectioning. The paraffin blocks were cut into $3 \mu \mathrm{m}$ sections, stained with Periodic Acid-Schiff (PAS), and used for mesangial analyses. Thirty glomeruli per animal were analyzed with a 20x objective lens by two independent, masked investigators. Mesangial expansion was evaluated using a semiquantitative scoring system as follows [22]: 0, no expansion; 1, expansion less than 25\%; 2, expansion between $25 \%$ and $50 \%$; 3 , expansion between $50 \%$ and $75 \%$; and 4 , expansion greater than $75 \%$ of the mesangial area.

2.5. Transmission Electron Microscopy (TEM). Kidney cortical tissue from each group was fixed with glutaraldehyde, embedded in Epon resin, and thinly sliced (60 to $100 \mathrm{~nm}$ ) using an ultramicrotome (Leica). Then, the ultrathin sections were collected on copper grids and stained with uranyl acetate and lead citrate. Finally, transmission electron microscopy was performed as previously described [23].

2.6. Immunohistochemistry (IHC). Murine renal tissue from each group was fixed for immunohistochemical staining in $10 \%$ neutral formalin, processed in the standard manner, and cut into $3 \mu \mathrm{m}$ sections. Slides were deparaffinized, hydrated in ethyl alcohol, and washed in tap water. Then, the slides underwent antigen retrieval and blocking with 5\% BSA. To assess RANKL, RANK, and nephrin in renal tissues, the sections were incubated with an anti-RANKL polyclonal antibody (RANKL, 1:50, Santa Cruz, CA, USA), an antiRANK polyclonal antibody (RANK, 1:50, Santa Cruz, CA, USA), or an anti-nephrin polyclonal antibody (nephrin, 1:50, Santa Cruz, CA, USA) overnight at $4^{\circ} \mathrm{C}$. After washing, a secondary donkey anti-rabbit antibody was added for 30 minutes $(\mathrm{min})$ at $37^{\circ} \mathrm{C}$; the slides were then washed and incubated with $\mathrm{DAB}$ for 2 min before counterstaining with hematoxylin. Sections were viewed and imaged with a light microscope (Ni-U, Nikon Corporation, Tokyo, Japan). Images were analyzed quantitatively by Image-Pro Plus 6.0 (IPP, Media Cybernetics, Inc., USA).

2.7. Enzyme-Linked Immunosorbent Assay. At the end of the experiment, urine samples from the mice were collected and centrifuged at $10,000 \times \mathrm{g}$ for $5 \mathrm{~min}$ at $4^{\circ} \mathrm{C}$ to remove the debris from the supernatants. Then, the supernatants were assayed using a solid-phase quantitative sandwich ELISA kit for MCP-1, as previously described [24]. The urinary MCP-1 concentration was normalized to the urinary creatinine concentration. 
2.8. Quantitative Real-Time PCR Analysis. RNA from the kidneys of each group of mice was extracted as previously described [21]. The primers for qPCR were as follows: MCP-1 forward: $5^{\prime}$-CCC AAT GAG TAG GCT GGA GA-3', reverse: $5^{\prime}$-TCT GGA CCC ATT CCT TCT TG-3', and GAPDH forward: $5^{\prime}$-ATT GTC AGC AAT GCA TCC TG-3', reverse: $5^{\prime}$-ATG GAC TGT GGT CAT GAG CC-3'. All data were normalized to GAPDH, and the expression levels were analyzed by the $2^{-\mathrm{DDCT}}$ method.

2.9. Western Blot Analysis. Total proteins were extracted from the kidneys, separated on $10 \%$ SDS-polyacrylamide gels, and transferred onto PVDF membranes (Millipore, Bedford, MA). The membrane was blocked for $1 \mathrm{~h}$ with Tris-buffered saline (20 mM Tris- $\mathrm{HCl}, 140 \mathrm{mM} \mathrm{NaCl}, \mathrm{pH}$ 7.6) containing $5 \%$ nonfat dry milk, washed with TBS containing $0.1 \%$ Tween-20, and incubated overnight at $4^{\circ} \mathrm{C}$ with a primary antibody. The primary RANKL rabbit $\mathrm{mAb}$ and RANK rabbit $\mathrm{mAb}$ were purchased from Santa Cruz Biotechnology (Santa Cruz, CA, USA). NF- $\kappa$ B p 65 rabbit mAb, p-NF- $\kappa$ B p 65 rabbit $\mathrm{mAb}, \mathrm{p}-\mathrm{I} \kappa \mathrm{B} \alpha$ rabbit $\mathrm{mAb}$, and $\mathrm{I} \kappa \mathrm{B} \alpha$ mouse $\mathrm{mAb}$ were purchased from Cell Signaling Technology (Danvers, MA). $\beta$ actin mouse $\mathrm{mAb}$ and HRP-conjugated secondary antibodies were purchased from EarthOx (EarthOx, LLC, San Francisco, CA, USA). The protein band intensities were quantified with Quantity One 4.6.2 analysis software (Quantity One, Bio-Rad Laboratories, Inc., USA), which was provided with the Kodak 2000MM System (Eastman Kodak Company, Rochester, New York, USA).

2.10. Statistical Analysis. The results are presented as the mean \pm S.D. Differences among multiple groups were analyzed by one-way ANOVA followed by a $t$-test to detect between-group differences. A two-sided $P<0.05$ was considered significant. All analyses were performed using IBM SPSS Statistics 20.0 (SPSS, Chicago, IL, USA).

\section{Results}

3.1. Effect of Irb on Metabolic Indices. At the end of the experiment, FBG, FINS, TC, TG, and body weight (BW) levels were significantly increased in the $\mathrm{db} / \mathrm{db}$ mice compared with the $\mathrm{db} / \mathrm{m}$ mice (Table 1 ). After 12 weeks of treatment with Irb, the TC and TG levels of the $\mathrm{db} / \mathrm{db}$ mice decreased from $5.21 \pm$ $0.72 \mathrm{mmol} / \mathrm{L}$ and $2.55 \pm 0.60 \mathrm{mmol} / \mathrm{L}$ to $3.12 \pm 0.93 \mathrm{mmol} / \mathrm{L}$ and $1.08 \pm 0.61 \mathrm{mmol} / \mathrm{L}$, respectively. However, there were no significant differences in the FBG, FINS, and BW levels between $\mathrm{db} / \mathrm{db}$ mice treated with and without Irb.

3.2. Irb Alleviated Diabetes-Induced Renal Dysfunction. Blood urea nitrogen (BUN), serum creatinine (Scr), and urinary albumin to creatinine ratio (ACR) were evaluated to assess the renal function of the mice. Both BUN and Scr were measured at the end of the experiment. ACR was detected at $8,12,16$, and 20 weeks of age. BUN, Scr, and ACR were significantly increased in the $\mathrm{db} / \mathrm{db}$ mice compared with the $\mathrm{db} / \mathrm{m}$ mice (Figures 1(a)-1(c)). With age, ACR was increased dramatically in $\mathrm{db} / \mathrm{db}$ mice but remained stable in $\mathrm{db} / \mathrm{m}$ mice
TABLE 1: Animal characteristics at the end of the experiment.

\begin{tabular}{lccc}
\hline & $\mathrm{db} / \mathrm{m}$ & $\mathrm{db} / \mathrm{db}$ & $\mathrm{db} / \mathrm{db}+\mathrm{Irb}$ \\
\hline FBG $(\mathrm{mmol} / \mathrm{L})$ & $6.15 \pm 0.96$ & $28.17 \pm 3.70^{*}$ & $25.51 \pm 4.06^{*}$ \\
FINS $(\mathrm{ng} / \mathrm{mL})$ & $1.53 \pm 0.50$ & $5.64 \pm 1.12^{*}$ & $5.14 \pm 1.03^{*}$ \\
TC $(\mathrm{mmol} / \mathrm{L})$ & $1.96 \pm 0.27$ & $5.21 \pm 0.72^{*}$ & $3.12 \pm 0.93^{\#}$ \\
TG $(\mathrm{mmol} / \mathrm{L})$ & $0.54 \pm 0.06$ & $2.55 \pm 0.60^{*}$ & $1.08 \pm 0.61^{\#}$ \\
BW $($ gram $)$ & $25.85 \pm 1.70$ & $46.61 \pm 3.31^{*}$ & $44.56 \pm 3.95^{*}$ \\
\hline
\end{tabular}

Data are the mean $\pm \mathrm{SD}, n=12$ per group. ${ }^{*} P<0.05$ versus $\mathrm{db} / \mathrm{m} .{ }^{\#} P<0.05$ versus $\mathrm{db} / \mathrm{db}$.

(Figure 1(c)). After 12 weeks of treatment with Irb, BUN and Scr were significantly decreased compared with the untreated $\mathrm{db} / \mathrm{db}$ mice (Figures 1(a) and 1(b)). Moreover, Irb markedly slowed the sharp ACR increase (Figure 1(c)). These results indicate that Irb can improve the renal function of $\mathrm{db} / \mathrm{db}$ mice.

\subsection{Irb Alleviated Diabetes-Induced Renal Pathological} Changes. At the end of the experiment, kidney tissues were collected for the PAS stain. The $\mathrm{db} / \mathrm{db}$ mice showed hyaline degeneration in the glomerular afferent arteries and mesangial expansion with major accumulation of mesangial matrix in the glomerulus (Figure 2(a)). The mesangial expansion score was significantly higher in $\mathrm{db} / \mathrm{db}$ mice than in $\mathrm{db} / \mathrm{m}$ mice, and this increase was reversed by Irb (Figure 2(b)).

3.4. Irb Alleviated Diabetes-Induced Podocyte Injury and Thickening of the GBM. To examine podocyte injury and the thickness of the GBM, kidney tissues were observed via TEM. Using electron microscopy, we noted that in $\mathrm{db} / \mathrm{db}$ mice, foot process effacement occurred in most of the podocytes, and the thickness of the GBM significantly increased compared with the $\mathrm{db} / \mathrm{m}$ mice (Figures 3(a) and 3(c)(A)). Moreover, nephrin, a marker of functional podocytes, was detected in kidney tissues by IHC. The results in Figure 3 show discontinuous punctiform or linear expression of nephrin in $\mathrm{db} / \mathrm{db}$ mice but continuous linear expression of nephrin in $\mathrm{db} / \mathrm{m}$ mice (Figure 3(b)). Compared with the $\mathrm{db} / \mathrm{m}$ mice, nephrin was reduced significantly in $\mathrm{db} / \mathrm{db}$ mice (Figures $3(\mathrm{~b})$ and $3(\mathrm{c})(\mathrm{B}))$. After 12 weeks of treatment with Irb, the podocyte injury was improved markedly; however, the thickness of the GBM did not change significantly between the $\mathrm{db} / \mathrm{db}$ mice treated with and without Irb (Figures 3(a)-3(c)).

3.5. Irb Attenuated Diabetes-Induced MCP-1 Expression. MCP-1 attracts macrophages and promotes inflammation in DN $[7,9,10]$. Thus, we examined the protein level of MCP-1 in the urine of mice and the mRNA level of MCP-1 in kidney tissues. As shown in Figure 4, the urinary concentrations of MCP-1 were significantly increased in $\mathrm{db} / \mathrm{db}$ mice compared with $\mathrm{db} / \mathrm{m}$ mice. After Irb treatment, the MCP-1 concentrations were drastically lowered in the urine (Figure 4(a)). Similarly, the MCP-1 mRNA level was also increased in the kidney of $\mathrm{db} / \mathrm{db}$ mice and was reduced by Irb treatment (Figure 4(b)). 


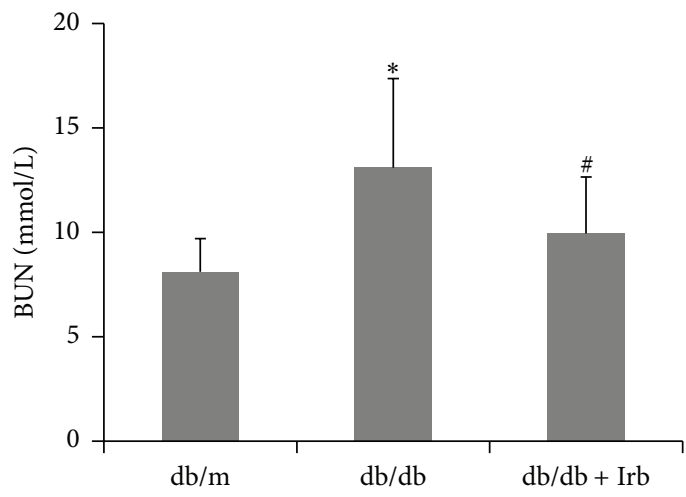

(a)

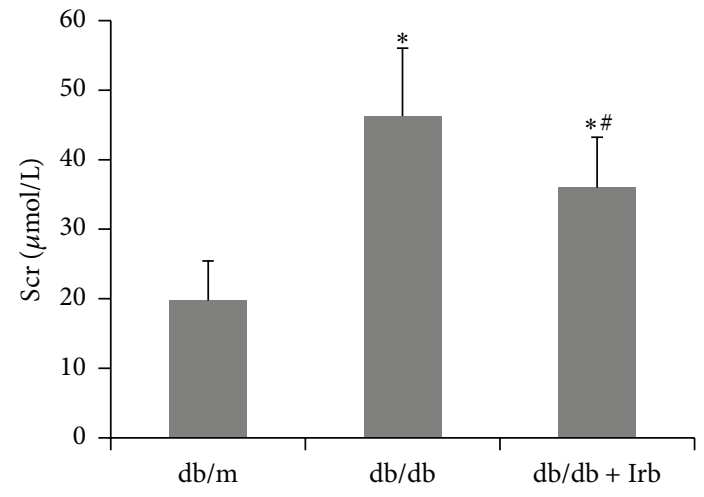

(b)

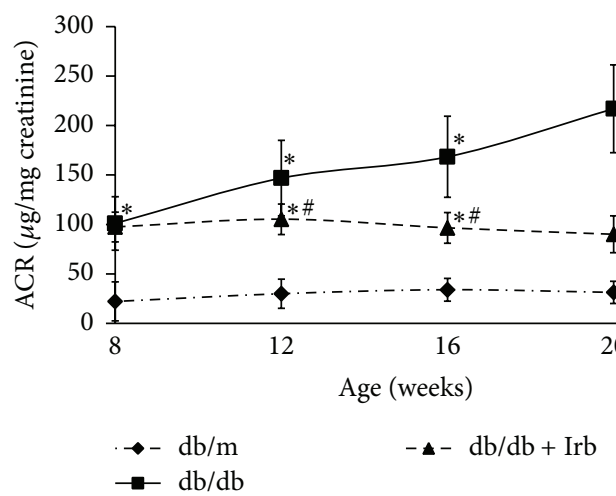

(c)

FIGURE 1: Irb alleviated diabetes-induced renal dysfunction. (a) BUN was increased in $\mathrm{db} / \mathrm{db}$ mice and reversed significantly by Irb. (b) Scr was increased in db/db mice and reversed significantly by Irb. (c) ACR increased dramatically in db/db mice in an age-dependent method, but this increase was reversed significantly by Irb. The bars in panel $(\mathrm{a}-\mathrm{c})$ show the mean expression level in arbitrary units (error bars, S.D.). ${ }^{*} P<0.05$ compared with $\mathrm{db} / \mathrm{m} ;{ }^{\#} P<0.05$ compared with $\mathrm{db} / \mathrm{db}, t$-test.
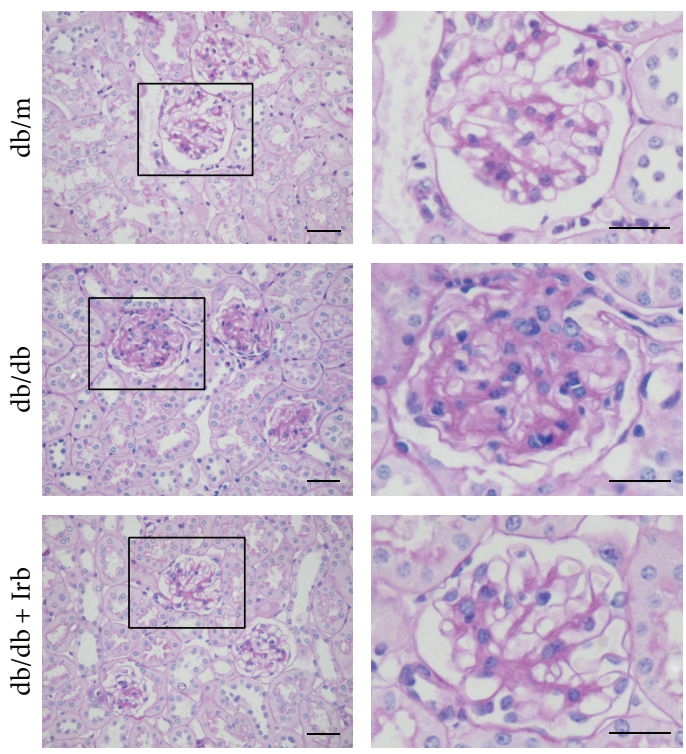

(a)

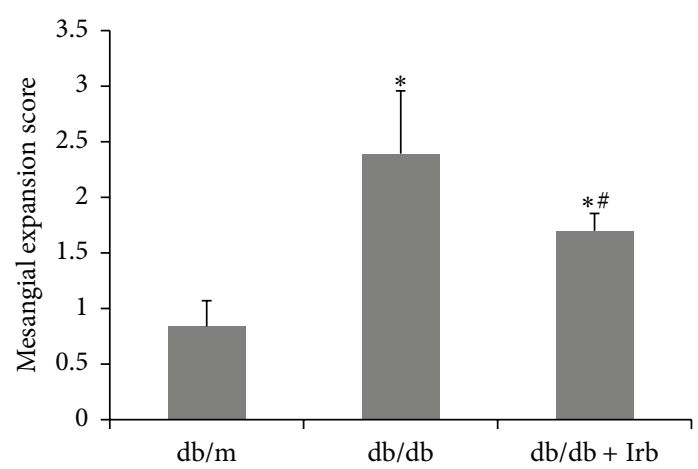

(b)

FIGURE 2: Irb alleviated diabetes-induced renal pathological changes. (a) Representative fields of the glomerulus stained with PAS (scale bars: $50 \mu \mathrm{m}$ ). (b) The mesangial expression score increased in $\mathrm{db} / \mathrm{db}$ mice and decreased in $\mathrm{db} / \mathrm{db}$ mice with Irb treatment. The bars in panel (b) show the mean expression in arbitrary units (error bars, S.D.). ${ }^{*} P<0.05$ compared with $\mathrm{db} / \mathrm{m} ;{ }^{\#} P<0.05$ compared with $\mathrm{db} / \mathrm{db}, t$-test. 


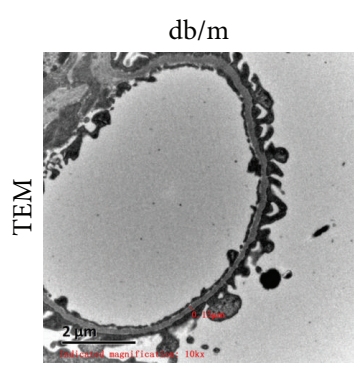

$\mathrm{db} / \mathrm{m}$
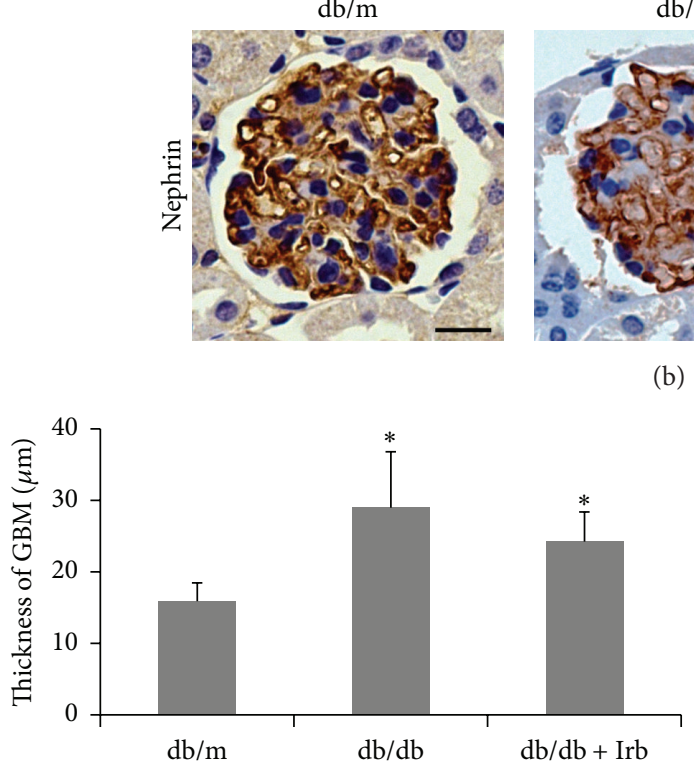

(A)

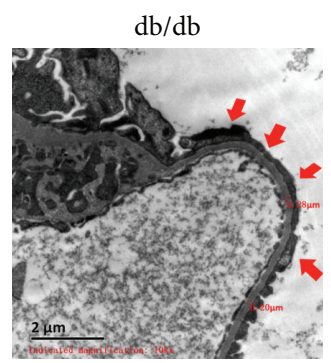

(a)

$\mathrm{db} / \mathrm{db}$

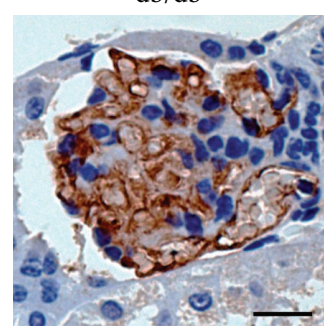

(b)

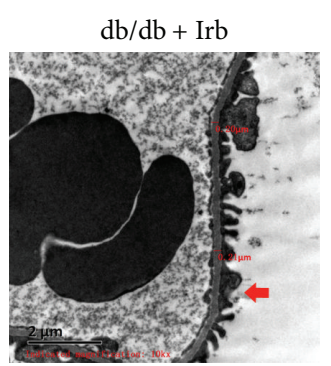

$\mathrm{db} / \mathrm{db}+\mathrm{Irb}$

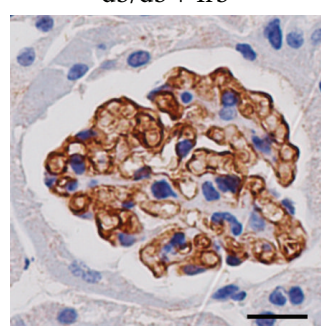

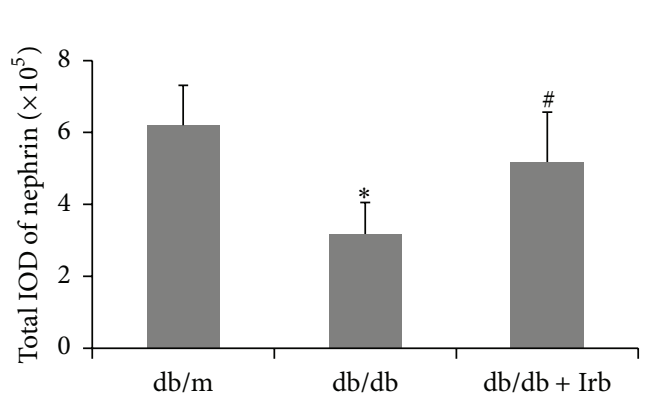

(B)

(c)

FIGURE 3: Irb alleviated diabetes-induced podocyte injury and the thickening of the GBM. (a) Representative fields of podocyte foot processes under TEM (scale bars: $2 \mu \mathrm{m}$, red arrow indicates podocyte foot process effacement). (b) Representative fields of nephrin, as labeled by immunohistochemical staining (scale bars: $50 \mu \mathrm{m}$ ). (c) Quantification of the GBM thickness (A) and immunohistochemical staining (B). The bars in panel (c) show the mean expression in arbitrary units (error bars, S.D.). ${ }^{*} P<0.05$ compared with db/m; ${ }^{\#} P<0.05$ compared with $\mathrm{db} / \mathrm{db}, t$-test.

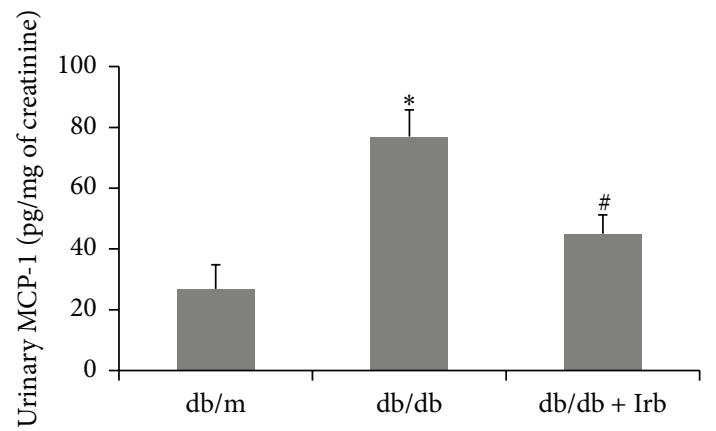

(a)

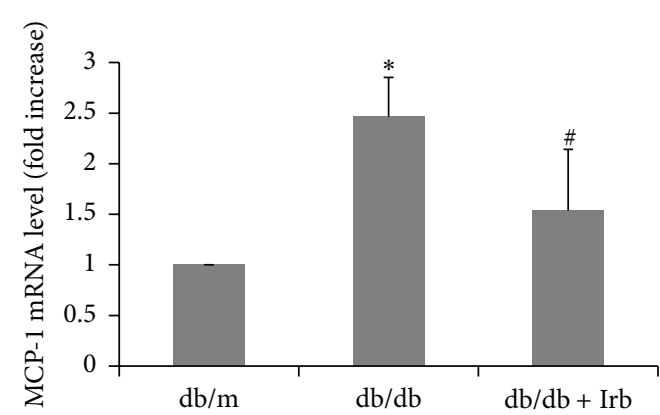

(b)

FIGURE 4: Irb attenuated diabetes-induced MCP-1 expression. (a) Urinary MCP-1 was significantly increased in db/db mice and significantly reduced in $\mathrm{db} / \mathrm{db}$ mice with treatment of Irb. (b) The mRNA level of MCP-1 in the kidney was increased in the db/db mice and reversed by Irb treatment significantly. The bars in panels (a) and (b) show the mean expression in arbitrary units (error bars, S.D.). ${ }^{*} P<0.05$ compared with $\mathrm{db} / \mathrm{m}$; ${ }^{\#} P<0.05$ compared with $\mathrm{db} / \mathrm{db}, t$-test. 
3.6. RANKL and RANK Were Overexpressed in $d b / d b$ Mice and Downregulated by Irb. We investigated whether RANKL and RANK were overexpressed in $\mathrm{db} / \mathrm{db}$ mice. As shown in Figure 5, db/db mice had a significantly increased level of immunohistochemical staining for RANKL and RANK compared with $\mathrm{db} / \mathrm{m}$ mice (Figures 5(a) and 5(c)(A)). We found that RANKL and RANK were mainly expressed in the glomeruli, along the glomerular capillary loop in $\mathrm{db} / \mathrm{db}$ mice. Moreover, the protein levels of RANKL and RANK were also significantly higher in the kidneys of $\mathrm{db} / \mathrm{db}$ mice (Figures 5(b) and 5(c)(B)). After 12 weeks of treatment with Irb, RANKL and RANK were downregulated (Figures 5(a)-5(c)).

3.7. Irb Inhibited NF- $\kappa B$ Pathway Activation in $d b / d b$ Mice. Compared with $\mathrm{db} / \mathrm{m}$ mice, $\mathrm{db} / \mathrm{db}$ mice showed significantly increased phosphorylation of $\mathrm{I} \kappa \mathrm{B} \alpha$ and $\mathrm{NF}-\kappa \mathrm{B} / \mathrm{p} 65$ and reduced $\mathrm{I} \kappa \mathrm{B} \alpha$ protein levels (Figures $6(\mathrm{a})$ and $6(\mathrm{~b})$ ), suggesting that the NF- $\kappa \mathrm{B}$ pathway is activated. After 12 weeks of treatment with Irb, NF- $\kappa$ B activation in the $\mathrm{db} / \mathrm{db}$ mice was blocked (Figures 6(a) and 6(b)).

\section{Discussion}

$\mathrm{DN}$ is a critical complication and a leading cause of ESRD in diabetes patients. Thus, a comprehensive understanding of the mechanisms underlying the pathogenesis of $\mathrm{DN}$ is urgently needed. Our present study investigated the role of RANKL and RANK in type $2 \mathrm{DN}$ and whether the beneficial effects of Irb in DN are mediated by the RANKL-RANKNF- $\kappa$ B pathway. The major findings of our study are that (1) RANKL and RANK are overexpressed in kidneys of mice with type $2 \mathrm{DN}$; (2) the NF- $\kappa \mathrm{B}$ pathway is activated in $\mathrm{DN}$; (3) Irb treatment alleviates diabetes-induced renal dysfunction, podocyte injury, and MCP-1 expression; and (4) the beneficial effects of Irb in DN are associated with its ability to suppress RANKL/RANK and the downstream NF- $\kappa$ B pathway. To the best of our knowledge, our study indicates, for the first time, that RANKL and RANK play important roles in the development of DN and that they are key targets of Irb treatment.

T2DM, the more prevalent form of diabetes in diabetic patients, is characterized by hyperglycemia, hyperlipidemia, and insulin resistance [25]. In our study, we used $\mathrm{db} / \mathrm{db}$ mice, which are a spontaneous T2DM mouse model, to explore the development and treatment of DN. At 8 weeks of age, db/db mice showed increased ACR, suggesting the occurrence of $\mathrm{DN}$. Then, we started to treat one group of the $\mathrm{db} / \mathrm{db}$ mice with Irb for 12 weeks. At the end of the experiment, the $\mathrm{db} / \mathrm{db}$ mice showed severe metabolic abnormalities, including elevated FBG, FINS, TG, TC, and BW. Additionally, as evaluated by renal function and histopathology analysis, our $\mathrm{db} / \mathrm{db}$ mice revealed overt DN, showing increased BUM, Scr, and ACR accompanied by mesangial expansion, podocyte foot process effacement, and thickening of the GBM.

In diabetes, the renin-angiotensin system (RAS) of the kidneys is activated. The RAS plays an important role in the regulation of systemic blood pressure. Angiotensin II, the final product of this system, which constricts blood vessels and raises blood pressure, mainly binds to angiotensin II type 1 receptor (AT1-R) and AT2-R [26]. Because the major pathogenic signaling of angiotensin II is mediated by AT1$\mathrm{R}, \mathrm{AT} 1-\mathrm{R}$ blockers (ARBs) are widely used in patients with hypertension and cardiovascular diseases. Moreover, accumulating evidence has suggested that ARBs have beneficial effects in patients with DN [11-15, 27, 28]. According to the available data from clinical trials, Irb, a widely used ARB in clinical practice, has a more pronounced pharmacological effect than other compounds within the same class. Based on two studies from the New England Journal of Medicine $[27,28]$, Irb is effective in treating both early and late stage nephropathy in hypertensive patients with T2DM. Additionally, the Irbesartan Diabetic Nephropathy Trial conducted by Lewis revealed that Irb slows the progression of nephropathy in patients with type 2 diabetes independently of its blood pressure-lowering effect. In animal studies, Tunçdemir and Öztürk [29] found that Irb exerts its renoprotective effects, including regulating renal hemodynamics and controlling tissue damage, by preventing podocyte loss. Bonnet et al. [30] investigated the effect of Irb on streptozotocin-induced diabetic spontaneously hypertension rats. They demonstrated the efficacy of Irb in reducing albuminuria, which was associated with the downregulation of nephrin at the gene and protein level. Data from Hartner et al. [15] study showed that low dose Irb increases synaptopodin, which is a marker of functional podocytes, and reduces desmin, which is a marker of podocyte damage in renal tissue at the protein level. These changes occurred in response to a reduction in blood pressure and inflammation but not a reduction of ER stress and apoptosis. In the present study, we found that Irb treatment ameliorated podocyte foot process effacement, upregulated the expression of nephrin, and decreased the level of MCP-1 in both urine and renal tissue, suggesting that Irb may exert its major renoprotective effects by preventing podocyte injury and controlling inflammation in the kidney.

To gain further insights into the potential mechanism involved in the renoprotective effect of Irb in DN, we focused on RANKL and RANK. The available evidence implicates RANKL and RANK as mediators of inflammation in T2DM [20]. Mice treated with hydrodynamic injection of Rank shRNA lentiviral vectors show inactivation of NF- $\kappa \mathrm{B}$ and the inhibition of downstream proinflammatory signaling in the liver. However, Liu et al. [18] reported that RANK and RANKL comprise a novel receptor-ligand pair in the survival response of injured podocytes. In their study, they found that the knockdown of RANK alone did not induce podocyte apoptosis but mildly and nonsignificantly increased the apoptosis of podocytes exposed to PAN. However, the application of exogenous RANKL significantly reduced the apoptosis of podocytes transfected with or without RANK siRNA when exposed to PAN. Their results show that RANK plays an insignificant role in the protection of podocytes and that RANKL reduces podocyte apoptosis independently of RANK. Thus, whether RANKL acts on RANK to protect podocytes from apoptosis remains controversial. In our opinion, RANKL may exert its antiapoptotic effect on PANinduced podocytes by binding the decoy receptor OPG, 

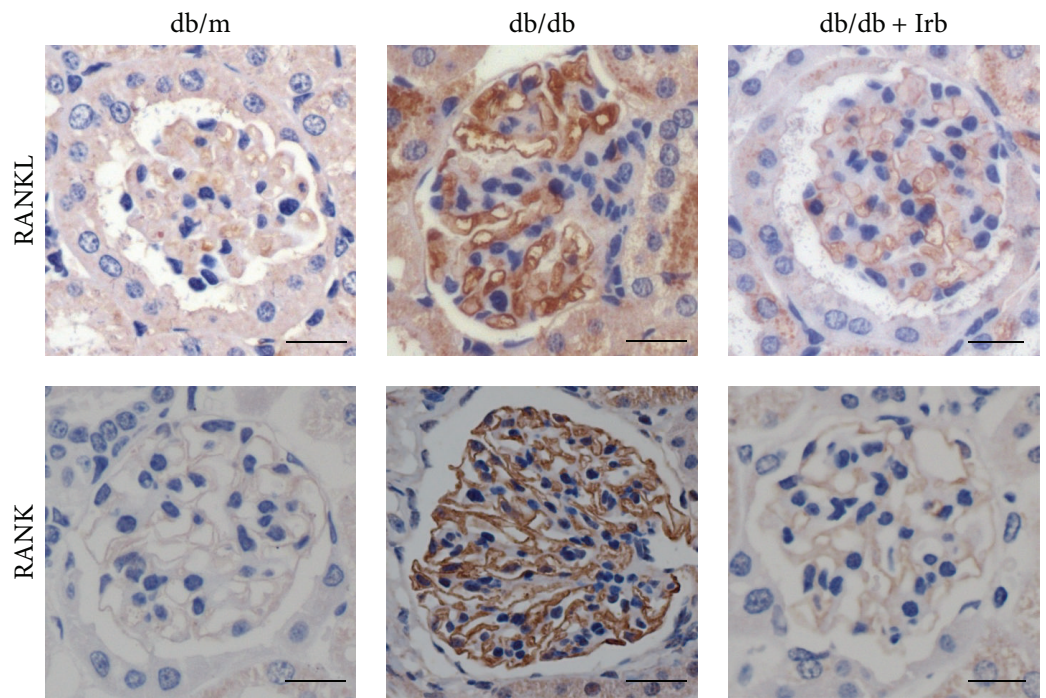

(a)

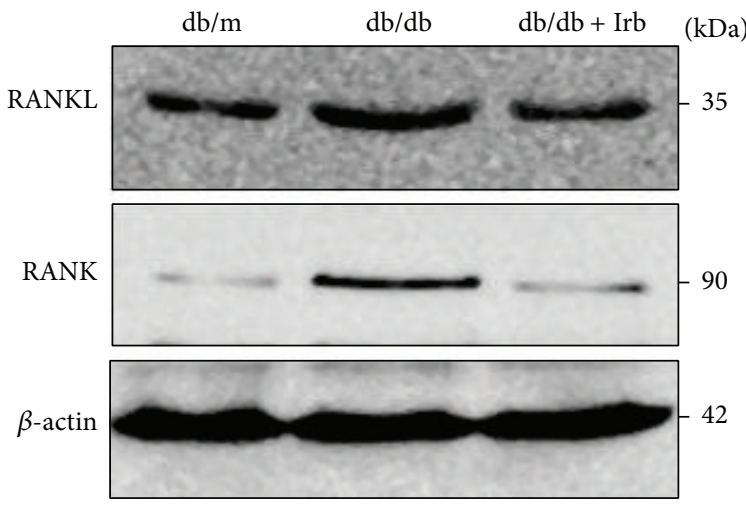

(b)

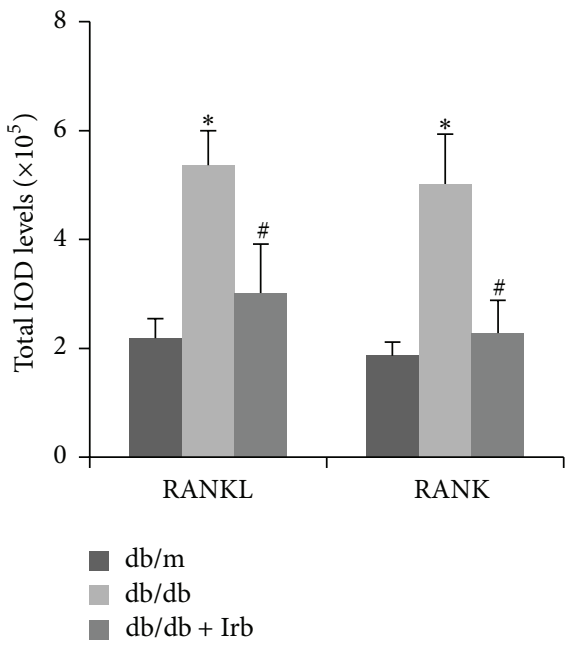

(A)

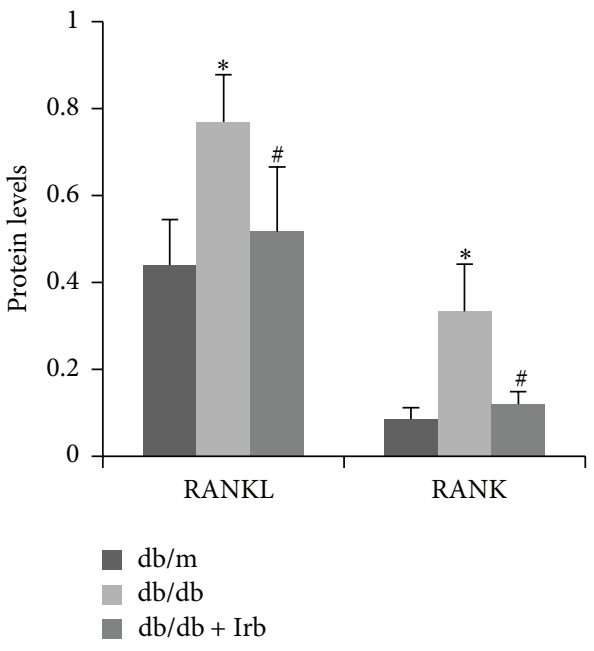

(B)

(c)

FIGURE 5: RANKL and RANK were overexpressed in $\mathrm{db} / \mathrm{db}$ mice and downregulated by Irb. (a) Representative fields of RANKL and RANK, as labeled by immunohistochemical staining (scale bars: $50 \mu \mathrm{m}$ ). (b) Representative immunoblots of RANKL and RANK expression in the kidney. (c) Quantification of the immunohistochemical staining (A). Quantification of the immunoblot (B): the ratio between RANKL and $\beta$-actin and the ratio between RANK and $\beta$-actin are presented. The bars in panel (c) show the mean expression in arbitrary units (error bars, S.D.). ${ }^{*} P<0.05$ compared with $\mathrm{db} / \mathrm{m} ;{ }^{\#} P<0.05$ compared with $\mathrm{db} / \mathrm{db}, t$-test. 

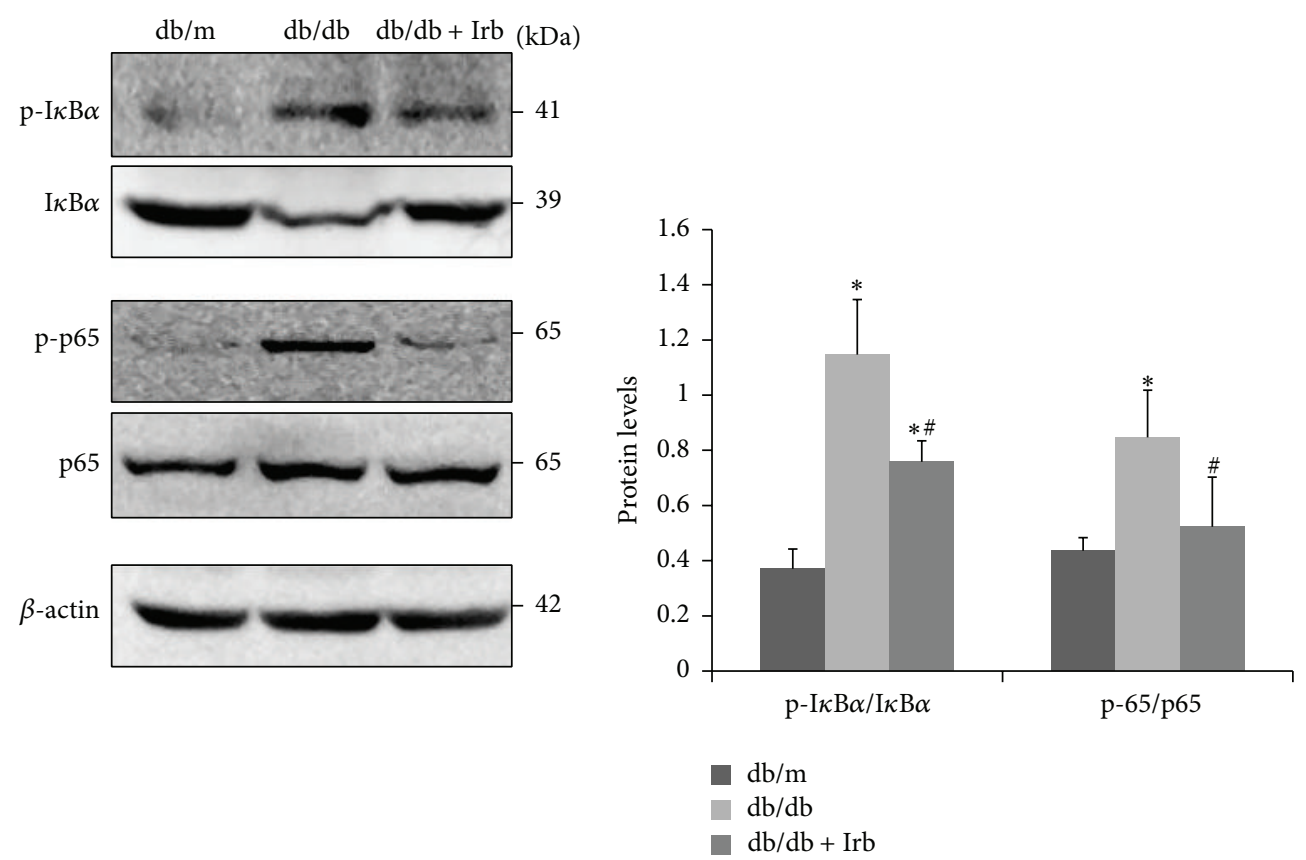

(a)

(b)

Figure 6: Irb inhibited NF- $\kappa \mathrm{B}$ pathway activation in $\mathrm{db} / \mathrm{db}$ mice. (a) Representative immunoblot of $\mathrm{p}-\mathrm{I} \kappa \mathrm{B} \alpha, \mathrm{I} \kappa \mathrm{B} \alpha$, $\mathrm{p}-\mathrm{p} 65$, and $\mathrm{p} 65$ in the kidney. (b) Quantification of the immunoblot: the ratio between $\mathrm{p}-\mathrm{I} \kappa \mathrm{B} \alpha$ and $\mathrm{I} \kappa \mathrm{B} \alpha$ and the ratio between p-p65 and p65 are presented. The bars in panel (b) show the mean expression in arbitrary units (error bars, S.D.). ${ }^{*} P<0.05$ compared with db/m; ${ }^{\#} P<0.05$ compared with $\mathrm{db} / \mathrm{db}, t$-test.

which acts as a survival factor for endothelial cells [17], pancreatic $\beta$-cells [31], and tubular cells [32,33]. When binding to RANK, RANKL activates the NF- $\kappa$ B pathway and subsequently invokes downstream proinflammatory signaling. Consistent with this pathway, our present results showed that RANKL and RANK were markedly increased in the kidneys of $\mathrm{db} / \mathrm{db}$ mice, and the NF- $\kappa \mathrm{B}$ pathway and its downstream inflammatory mediator MCP-1 were subsequently activated. Irb treatment reduced the elevated RANKL and RANK and inhibited activation of the NF- $\kappa$ B pathway.

In the present study, we demonstrated the renoprotective effects of irbesartan on DN. However, some limitations of our study are noted. First, the spontaneous T2DM db/db mouse model, which shares many metabolic phenotypes and renal abnormalities that are observed in patients, may still have some limited relevance to clinical conditions. Second, our study demonstrated the involvement of RANKL and RANK in the development of DN. However, the precise role of RANKL and RANK in diabetes-induced renal dysfunction and podocyte injury has not been definitively verified. Further, in vivo experiments employing RANKspecific inhibitors or RANK gene silencing are preferential to clarify the underlying mechanism.

\section{Conclusions}

In conclusion, Irb alleviated renal dysfunction, podocyte injury, and MCP-1 expression in DN. The molecular mechanism responsible for the protective effects of Irb may involve inhibition of the RANKL-RANK-NF- $\kappa$ B pathway. Our study provides a novel mechanism through which Irb treats DN.

\section{Conflict of Interests}

The authors have no conflict of interests to declare.

\section{Authors' Contribution}

Hai-Bo Long and Xiao-Wen Chen conceived and designed the experiments. Xiao-Wen Chen, Xiao-Yan Du, Yu-Xian Wang, Jian-Cheng Wang, Wen-Ting Liu, Wen-Jing Chen, Hong-Yu Li, Fen-Fen Peng, Zhao-Zhong Xu, Hong-Xin Niu, and Hai-Bo Long performed the experiments and analyzed the data. Hai-Bo Long and Xiao-Wen Chen wrote the paper. All authors read and approved the final paper. Xiao-Wen Chen and Xiao-Yan Du contributed equally to this study.

\section{Acknowledgments}

This work was supported by the National Natural Science Foundation of China (NSFC) (no. 81072848 to Hai-Bo Long and 81170682 to Hong-Xin Niu), the Science and Technology Planning Project of Guangdong Province, China (nos. 2013B021800149, 2014A020210011, and 2015A020211012 to Hai-Bo Long), the Natural Science Foundation of Guangdong Province, China (no. 2014A030310065 to Fen-Fen Peng), and the Science and Technology Planning Project of Guangzhou, China (no. 201510010137 to Hai-Bo Long). 


\section{References}

[1] E. Ritz and S. R. Orth, "Nephropathy in patients with type 2 diabetes mellitus," The New England Journal of Medicine, vol. 341, no. 15, pp. 1127-1133, 1999.

[2] E. Ritz, I. Rychlik, F. Locatelli, and S. Halimi, "End-stage renal failure in type 2 diabetes: a medical catastrophe of worldwide dimensions," American Journal of Kidney Diseases, vol. 34, no. 5, pp. 795-808, 1999.

[3] A. R. Andersen, J. S. Christiansen, J. K. Andersen, S. Kreiner, and T. Deckert, "Diabetic nephropathy in type 1 (insulindependent) diabetes: an epidemiological study," Diabetologia, vol. 25, no. 6, pp. 496-501, 1983.

[4] G. L. Bakris, M. Williams, L. Dworkin et al., "Preserving renal function in adults with hypertension and diabetes: a consensus approach," American Journal of Kidney Diseases, vol. 36, no. 3, pp. 646-661, 2000.

[5] T. W. Meyer, P. H. Bennett, and R. G. Nelson, "Podocyte number predicts long-term urinary albumin excretion in Pima Indians with Type II diabetes and microalbuminuria," Diabetologia, vol. 42, no. 11, pp. 1341-1344, 1999.

[6] P. Anil Kumar, G. I. Welsh, M. A. Saleem, and R. K. Menon, "Molecular and cellular events mediating glomerular podocyte dysfunction and depletion in diabetes mellitus," Frontiers in Endocrinology, vol. 5, article 151, 2014.

[7] F. Chow, E. Ozols, D. J. Nikolic-Paterson, R. C. Atkins, and G. $\mathrm{H}$. Tesch, "Macrophages in mouse type 2 diabetic nephropathy: correlation with diabetic state and progressive renal injury," Kidney International, vol. 65, no. 1, pp. 116-128, 2004.

[8] W. H. Yiu, M. Lin, and S. C. W. Tang, "Toll-like receptor activation: from renal inflammation to fibrosis," Kidney International Supplements, vol. 4, no. 1, pp. 20-25, 2014.

[9] H. Kanamori, T. Matsubara, A. Mima et al., "Inhibition of MCP-1/CCR2 pathway ameliorates the development of diabetic nephropathy," Biochemical and Biophysical Research Communications, vol. 360, no. 4, pp. 772-777, 2007.

[10] B. Amann, R. Tinzmann, and B. Angelkort, "ACE inhibitors improve diabetic nephropathy through suppression of renal MCP-1," Diabetes Care, vol. 26, no. 8, pp. 2421-2425, 2003.

[11] X. Ren, G. Guan, G. Liu, and G. Liu, "Irbesartan ameliorates diabetic nephropathy by reducing the expression of connective tissue growth factor and alpha-smooth-muscle actin in the tubulointerstitium of diabetic rats," Pharmacology, vol. 83, no. 2, pp. 80-87, 2009.

[12] C. Wang, C. Min, X. Rong, T. Fu, X. Huang, and C. Wang, "Irbesartan can improve blood lipid and the kidney function of diabetic nephropathy," Discovery Medicine, vol. 20, no. 108, pp. 67-77, 2015.

[13] S.-I. Masuda, K. Tamura, H. Wakui et al., "Effects of angiotensin II type 1 receptor blocker on ambulatory blood pressure variability in hypertensive patients with overt diabetic nephropathy," Hypertension Research, vol. 32, no. 11, pp. 950-955, 2009.

[14] M. Nakamura, N. Sasai, I. Hisatome, and K. Ichida, "Effects of irbesartan on serum uric acid levels in patients with hypertension and diabetes," Clinical Pharmacology: Advances and Applications, vol. 6, no. 1, pp. 79-86, 2014.

[15] A. Hartner, N. Cordasic, B. Klanke et al., "Renal protection by low dose irbesartan in diabetic nephropathy is paralleled by a reduction of inflammation, not of endoplasmic reticulum stress," Biochimica et Biophysica Acta, vol. 1842, no. 4, pp. 558$565,2014$.
[16] S. Chen, G. Jin, K.-M. Huang et al., "Lycorine suppresses ranklinduced osteoclastogenesis in vitro and prevents ovariectomyinduced osteoporosis and titanium particle-induced osteolysis in vivo," Scientific Reports, vol. 5, Article ID 12853, 2015.

[17] U. M. Malyankar, M. Scatena, K. L. Suchland, T. J. Yun, E. A. Clark, and C. M. Giachelli, "Osteoprotegerin is an $\alpha(\mathrm{v}) \beta 3$ induced, NF- $\kappa \mathrm{B}$-dependent survival factor for endothelial cells," The Journal of Biological Chemistry, vol. 275, no. 28, pp. 20959-20962, 2000.

[18] S. Liu, W. Shi, H. Xiao et al., "Receptor activator of NF-kappaB and podocytes: towards a function of a novel receptor-ligand pair in the survival response of podocyte injury," PLOS ONE, vol. 7, no. 7, Article ID e41331, 2012.

[19] A. Leiherer, A. Muendlein, P. Rein et al., "Genome-wide association study reveals a polymorphism in the podocyte receptor RANK for the decline of renal function in coronary patients," PLoS ONE, vol. 9, no. 12, Article ID el14240, 2014.

[20] S. Kiechl, J. Wittmann, A. Giaccari et al., "Blockade of receptor activator of nuclear factor- $\kappa \mathrm{B}$ (RANKL) signaling improves hepatic insulin resistance and prevents development of diabetes mellitus," Nature Medicine, vol. 19, no. 3, pp. 358-363, 2013.

[21] X. Zhong, A. C. K. Chung, H. Y. Chen et al., "MiR-21 is a key therapeutic target for renal injury in a mouse model of type 2 diabetes," Diabetologia, vol. 56, no. 3, pp. 663-674, 2013.

[22] X. Bai, X. Li, J. Tian, and Z. Zhou, "Antiangiogenic treatment diminishes renal injury and dysfunction via regulation of local akt in early experimental diabetes," PLoS ONE, vol. 9, no. 4, Article ID e96117, 2014.

[23] E. Gagliardini, S. Conti, A. Benigni, G. Remuzzi, and A. Remuzzi, "Imaging of the porous ultrastructure of the glomerular epithelial filtration slit," Journal of the American Society of Nephrology, vol. 21, no. 12, pp. 2081-2089, 2010.

[24] Y. Zhao, S.-J. Chen, J.-C. Wang et al., "Sesquiterpene lactones inhibit advanced oxidation protein product-induced MCP-1 expression in podocytes via an IKK/NF- $\kappa \mathrm{B}$-dependent mechanism," Oxidative Medicine and Cellular Longevity, vol. 2015, Article ID 934058, 13 pages, 2015.

[25] Z.-H. Yang and X.-D. Peng, "Insulin resistance and heart injury in rats with insulin resistance or type 2 diabetes mellitus," Acta Cardiologica, vol. 65, no. 3, pp. 329-335, 2010.

[26] M. de Gasparo, K. J. Catt, T. Inagami, J. W. Wright, and T. Unger, "International union of pharmacology. XXIII. The angiotensin II receptors," Pharmacological Reviews, vol. 52, no. 3, pp. 415472, 2000.

[27] H.-H. Parving, H. Lehnert, J. Brochner-Mortensen, R. Gomis, S. Andersen, and P. Arner, "The effect of irbesartan on the development of diabetic nephropathy in patients with type 2 diabetes," The New England Journal of Medicine, vol. 345, no. 12, pp. 870-878, 2001.

[28] E. J. Lewis, L. G. Hunsicker, W. R. Clarke et al., "Renoprotective effect of the angiotensin-receptor antagonist irbesartan in patients with nephropathy due to type 2 diabetes," The New England Journal of Medicine, vol. 345, no. 12, pp. 851-860, 2001.

[29] M. Tunçdemir and M. Öztürk, "The effects of angiotensin-II receptor blockers on podocyte damage and glomerular apoptosis in a rat model of experimental streptozotocin-induced diabetic nephropathy," Acta Histochemica, vol. 113, no. 8, pp. 826-832, 2011.

[30] F. Bonnet, M. E. Cooper, H. Kawachi, T. J. Allen, G. Boner, and Z. Cao, "Irbesartan normalises the deficiency in glomerular nephrin expression in a model of diabetes and hypertension," Diabetologia, vol. 44, no. 7, pp. 874-877, 2001. 
[31] J. Schrader, W. Rennekamp, U. Niebergall et al., "Cytokineinduced osteoprotegerin expression protects pancreatic beta cells through p38 mitogen-activated protein kinase signalling against cell death," Diabetologia, vol. 50, no. 6, pp. 1243-1247, 2007.

[32] C. Lorz, A. Benito-Martin, A. Boucherot et al., "The death ligand TRAIL in diabetic nephropathy," Journal of the American Society of Nephrology, vol. 19, no. 5, pp. 904-914, 2008.

[33] R. Candido, "The osteoprotegerin/tumor necrosis factor related apoptosis-inducing ligand axis in the kidney," Current Opinion in Nephrology and Hypertension, vol. 23, no. 1, pp. 69-74, 2014. 


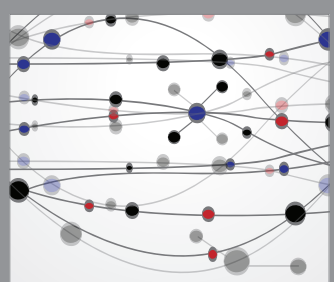

The Scientific World Journal
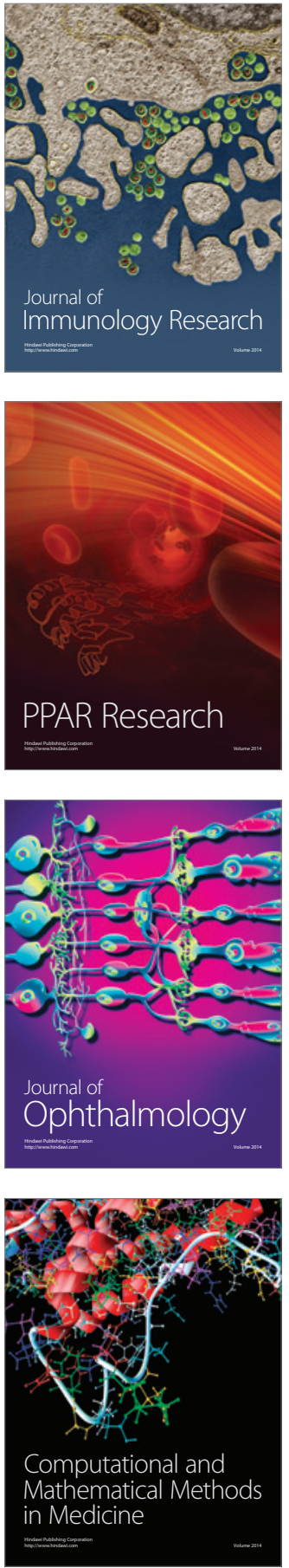

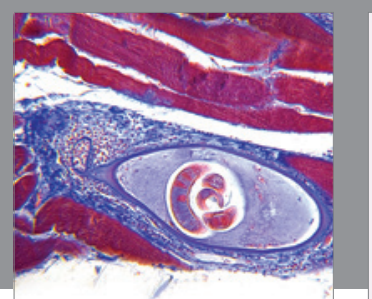

Gastroenterology Research and Practice

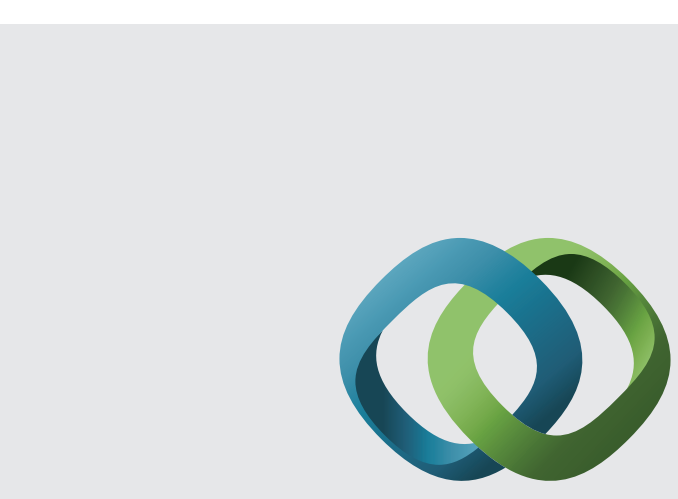

\section{Hindawi}

Submit your manuscripts at

http://www.hindawi.com
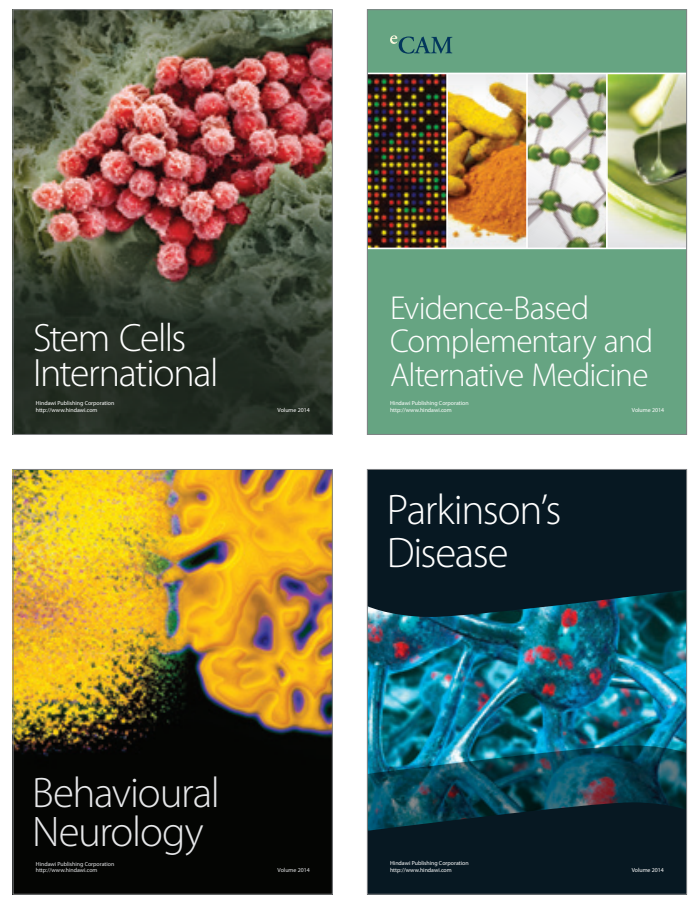
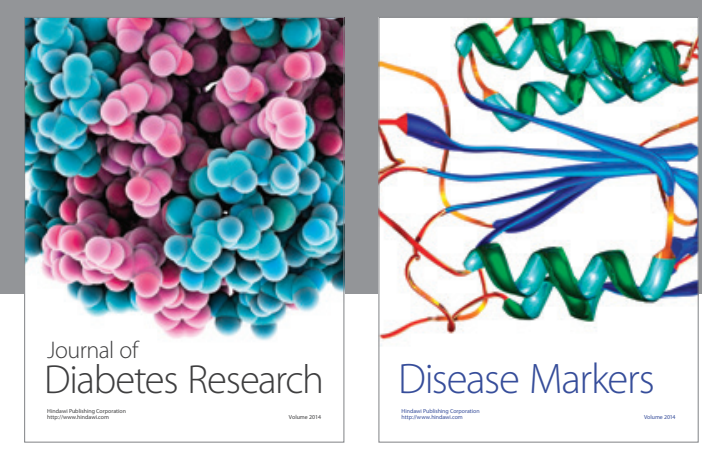

Disease Markers
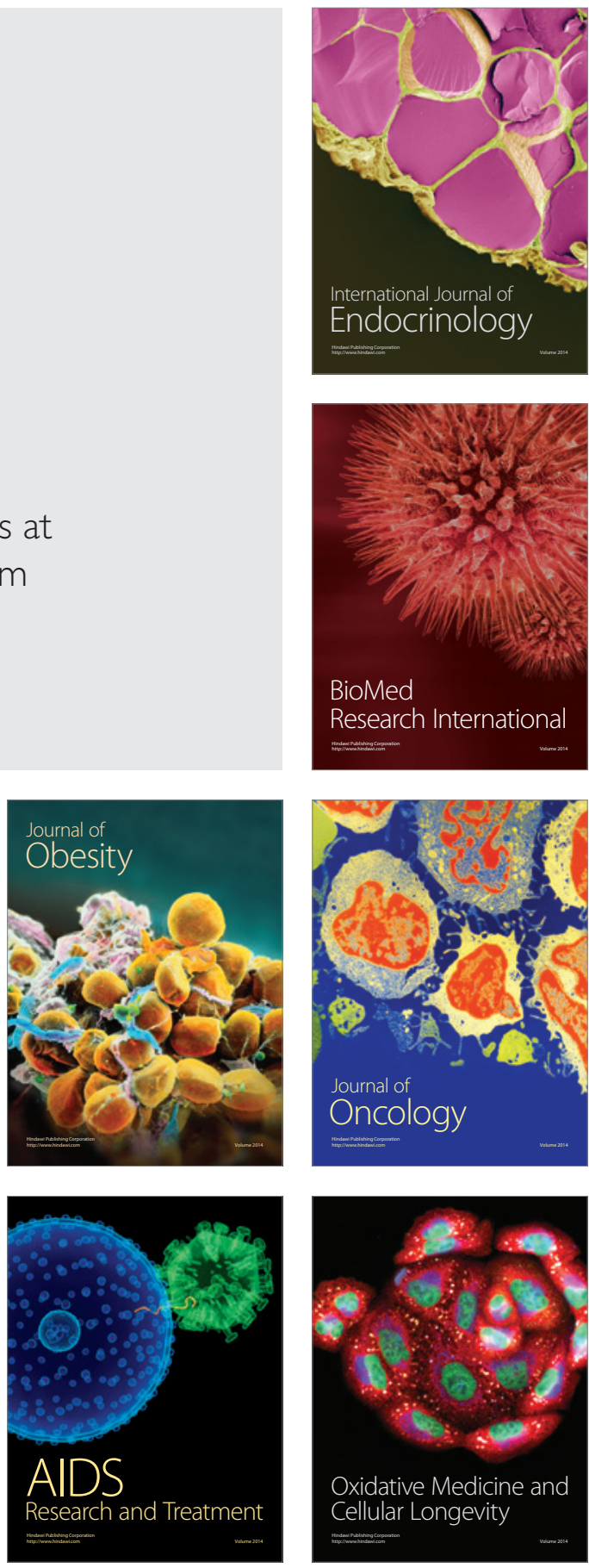